\title{
Detection of CAP59 Gene in 2 Feline Cases of Systemic Cryptococcosis
}

\author{
Ken OKABAYASHI ${ }^{1}$, Rui KANO ${ }^{1)}$, Kumiko SIROUZU ${ }^{2)}$, Tokuma YANAI'), Masao MIZUNO ${ }^{3)}$, \\ Hozumi YAMAMURA ${ }^{3)}$, Sanae SAEGUSA ${ }^{3)}$ and Atsuhiko HASEGAWA ${ }^{1)}$ \\ ${ }^{1)}$ Department of Pathobiology, Nihon University School of Veterinary Medicine, 1866 Kameino, Fujisawa Kanagawa, 252-8510, \\ ${ }^{2)}$ Department of Veterinary Pathology, Faculty of Agriculture, Gifu University, 1-1 Yanagido, Gifu 501-1193 and ${ }^{33}$ Kitagawa Animal \\ Hospital, 1-39-1 Minamitokiwadai, Itabashi-ku, Tokyo 174-0072, Japan
}

(Received 20 January 2003/Accepted 2 May 2003) ABSTRACT. Two feline cases were diagnosed as systemic cryptococcosis due to Cryptococcus neoformans (teleomorph: Filobasidiella neo-
formans) by PCR assay with CAP59 gene primers using urine, serum and biopsy samples. The results of molecular analysis were con-
sistent with the mycological findings.

KEY WORDS: CAP59 gene, feline, systemic cryptococcosis.

J. Vet. Med. Sci. 65(8): 953-955, 2003

Cryptococcosis is the most common systemic fungal infection in cats and should be considered in a differential diagnosis of cats with respiratory tract disease, subcutaneous nodules, lymphadenopathy, intraocular inflammation, fever, or CNS diseases [2, 4]. In recent years, molecular techniques have greatly improved diagnosis of human cryptococcal infections $[8,9]$, however the detection of the specific cryptococcal DNA by polymerase chain reaction (PCR) directly in clinical samples has not been well described in animal cryptococcosis. In a previous study, a PCR assay was developed specifically to detect Cryptococcus neoformans (teleomorph: Filobasidiella neoformans) in biopsy samples from cutaneous cryptococcosis in a cat [5]. However, the molecular diagnosis method has not been investigated in systemic cryptococcosis in cats. In this study, a PCR assay was carried out for 2 feline cases of systemic cryptococcosis.

Case 1, a 4-year-old female domestic cat, had many subcutaneous nodules on the head and the dorsum of the body. The cat had received antibiotic and steroid therapy. However, these nodules did not reduce, and lameness of the right hind leg appeared. The cat died without showing any improvement and was autopsied immediately at the Department of Veterinary Pathology of Gifu University. Post mortem examination revealed that many miliary nodules $(1 \mathrm{~mm}$ to $10 \mathrm{~mm}$ in diameter) were found in the subcutis, muscles of thorax and abdomen, abdominal cavity, liver, spleen, cecum, colon, kidneys, heart and lungs.

Case 2, a 19-year-old female domestic cat was admitted with the chief complaints of dermatitis and blindness. The cat had been treated with glucocorticoid therapy for a long time. The cat was diagnosed as systemic cryptococcosis by cytologic examination of the nodules and serologic test with PASTOREX CRYPTO PLUS (Sanofi Diagnostics Pasteur, Marnes-la-Co-quette, France), which detects the cryptococcal capsule antigen. The cat was treated with itraconazole

\footnotetext{
* Correspondence to: Dr. Kano, R., Department of Pathobiology, Nihon University School of Veterinary Medicine, 1866, Kameino, Fujisawa Kanagawa, 252-8510, Japan.
}

and fluconazole, and improved except for the optical abnormality.

The spleen and skin specimens from Case 1 and urine sample from Case 2 were cultured on sunflower seed agar at $25^{\circ} \mathrm{C}$ for 5 days. Each clinical isolate from the cases were dark-brown-colored, smooth, mucoide colonies. Microscopic examination of these clinical isolates revealed globose, ovoidal, or apiculate yeast cells. These yeast cells were encapsulated and some were budding. Their phenol oxidase activity and urease reaction were positive. The isolates were shown to be serotype A by serologic test with Crypto Check (Iatron Lab, Tokyo, Japan). From these results, the isolates were identified as Cryptococcus neoformans var. neoformans (teleomorph: Filobasidiella neoformans var. neoformans), serotype A.

The samples for molecular analyses were approximately $100 \mathrm{mg}$ biopsied spleen and skin tissues from Case 1, and $400 \mu l$ urine and serum from Case 2. DNA extraction from the biopsy specimens, urine and serum samples were prepared by the method reported previously [3].

The PCR assay was described in a previous report [3]. The pair of primers for for CAP59 gene were based on the sequences reported [6]: primer S-2, 5'-GAG TGT CTC CGC AAC CCG CA-3'; primer S-2, 5'-CCT ACT CTG CCA AAT CAA CTC-3'. The PCR amplification was carried out for 35 cycles consisting of template denaturation (1 $\mathrm{min}$, at $\left.94^{\circ} \mathrm{C}\right)$, primer annealing $\left(2 \mathrm{~min}\right.$, at $\left.60^{\circ} \mathrm{C}\right)$ and polymerization $\left(3 \mathrm{~min}\right.$, at $\left.72^{\circ} \mathrm{C}\right)$. Each PCR product was gelpurified and cloned into pCRII vector (Invitrogen, California, U.S.A.). Each plasmid DNA was extracted with Quantum Prep Plasmid mini Prep Kit (BIO-RAD, California, U.S.A.) and sequenced by the dideoxy chain termination method using ABI PRISM 310 Genetic Analyzer (Applied Biosystems, California, U.S.A.).

To examine homology relationships among the CAP59 genes of reference strains of $C$. neoformans as well as the samples, we used Clustal W multiple sequence alignment programs [10], and phylogenic trees were constructed by the TREEVIEW phylogeny display program [7]. Bootstrap 


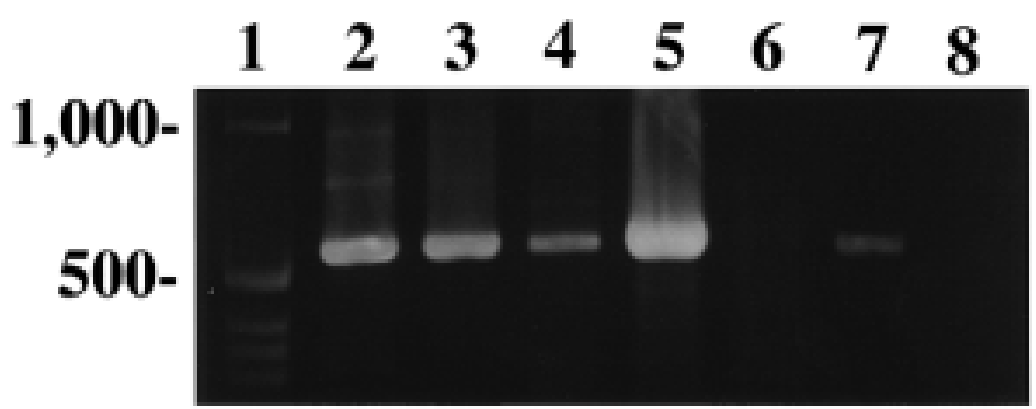

Fig. 1. Electrophoretic analysis of PCR products from genomic DNA samples amplified with CAP59 primer pair. Lane 1: DNA ladder marker. Lane 2: biopsied spleen of Case 1. Lane 3: skin tissue of Case 1. Lane 4: clinical isolate from urine sample of Case 2 cultured on sunflower seed agar for 5 days. Lane 5: urine sample of Case 2. Lane 6: urine sample of a healthy cat. Lane 7: serum sample of Case 2. Lane 8: serum sample of a healthy cat.

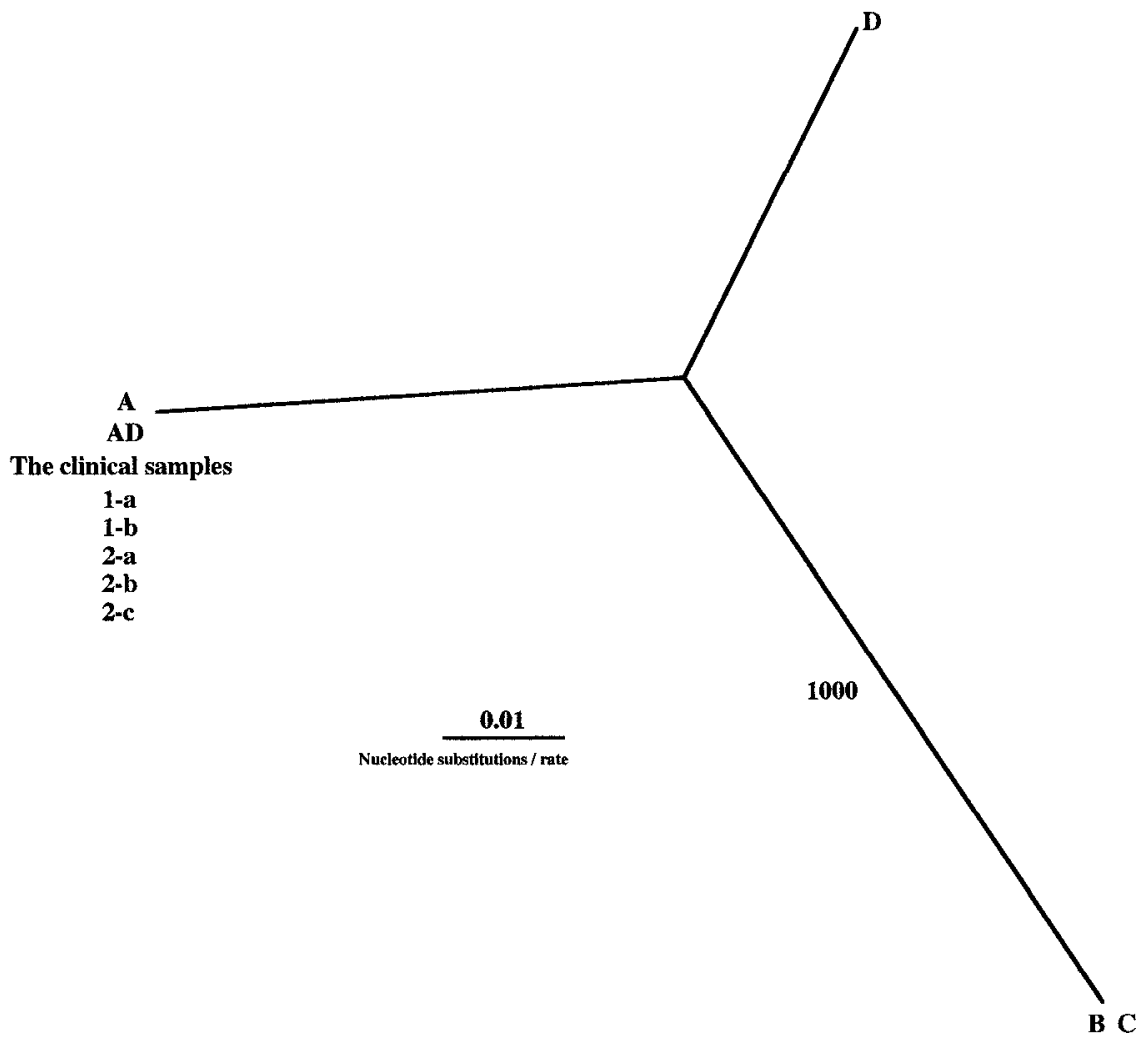

Fig. 2. A tree showing the phylogenetic relationships of CAP59 gene fragments of reference strains of C. neoformans as well as the clinical samples. 1-a: biopsied spleen of Case 1. 1-b: skin tissue of Case 1. 2-a: clinical isolate from urine sample of Case 2 cultured on sunflower seed agar for 5 days. 2-b: urine sample of Case 2. 2-c: serum sample of Case 2.

analyses were performed on 1,000 random samples taken from multiple alignment as described by Felsenstein [1].

Amplification of the samples from the cases with CAP59 primers yielded a 597-bp fragment (Fig. 1), consistent with the sizes of CAP59 gene sequences from fungal species reported previously [6]. The CAP59 nucleotide sequences from the clinical samples as well as the isolates showed more than $98 \%$ similarity to sequences of serotype A refer- ence strains (DDBJ accession no. AB019367), less than $90 \%$ similarity to serotype B (DDBJ accession no. AB019369) or C (DDBJ accession no. AB019370), and approximately $92 \%$ similarity to serotype D (DDBJ accession no. L26508). In addition, the phylogenetic analyses of clinical samples belonged to the cluster of serotype A and $\mathrm{AD}$ of the clusters reported previously (Fig. 2) [6].

The results of the molecular analysis revealed that these 
cases were infected with Cryptococcus neoformans var. neoformans, serotype A, consistent with the results of mycological analysis.

Since cryptococcosis is the most common systemic mycosis in cats, a rapid method of diagnosis is needed. The cytologic evaluation is the most rapid method but is quite an insensitive test and strongly depends on the operator's skills. The latex agglutination test is a more sensitive method but may still yield false-positive and false-negative results [3]. In a previous study [5], the molecular technique greatly improved diagnosis of feline cutaneous cryptococcal infections, however detection of disseminated feline cryptococcosis was not carried out. In this study, we investigated disseminated feline cryptococcosis by the PCR technique, which detected Cryptococcus DNA in serum and urine. These results demonstrate that the PCR detection system using genome DNA directly extracted from clinical samples could take a shorter time and be helpful for the rapid diagnosis of feline cryptococcosis.

\section{REFERENCES}

1. Felsenstein, J. 1985. Evolution 39: 783-791.

2. Flatland, B., Greene, R. T. and Lappin, M. R. 1996. J. Am. Vet. Med. Assoc. 209: 1110-1113.

3. Kano, R., Nakamura, Y., Watanabe, S. and Hasegawa, A. 2000. Micobiol. Immunol. 44: 605-607.

4. Kano, R., Nakamura, Y., Watari, T., Tsujimoto, H. and Hasegawa, A. 1997. Mycoses 40: 381-383.

5. Kano, R., Fujino Y., Takamoto, N., Tsujimoto, H. and Hasegawa, A. 2001. J. Vet. Diagn. Invest. 13: 439-442.

6. Nakamura, Y., Kano, R., Watanabe, S. and Hasegawa, A. 2000. J. Clin. Microbiol. 38: 992-995.

7. Page, R. D. 1996. Comput. Appl. Biosci. 12: 357-358.

8. Rappelli, P., Are, R., Casu, G., Fiori, P. L., Cappuccinell, P. and Aceti, A. 1998. J. Clin. Microbiol. 36: 3438-3440.

9. Tanaka, K., Miyazaki, T., Maesaki, S., Mitsutake, K., Kakeya, H., Yamamoto, Y., Yanagihara, K., Hossain, M. A., Tashiro, T. and Kohno, S. 1996. J. Clin. Microbiol. 34: 2826-2828.

10. Thompson, J. D., Higgins, D. G. and Gibson, T. J. 1994. Nuc. Acid. Res. 22: 4673-4680. 